\title{
Attention Enhances the Efficacy of Communication in V1 Local Circuits
}

\author{
Jacqueline R. Hembrook-Short, ${ }^{1}$ Vanessa L. Mock, ${ }^{1,2,3} \mathbb{C}^{-W}$. Martin Usrey, ${ }^{4}$ and ${ }^{\oplus}$ Farran Briggs ${ }^{3,5,6,7}$ \\ ${ }^{1}$ Physiology \& Neurobiology Department, Geisel School of Medicine at Dartmouth, Lebanon, New Hampshire 03756, ${ }^{2}$ Program in Experimental and \\ Molecular Medicine, Dartmouth College, Hanover, New Hampshire 03755, ${ }^{3}$ Ernest J. Del Monte Institute for Neuroscience, University of Rochester School \\ of Medicine, Rochester, New York 14642, ${ }^{4}$ Center for Neuroscience, University of California at Davis, Davis, California 95618, ${ }^{5}$ Department of Neuroscience, \\ University of Rochester School of Medicine, Rochester, New York 14642, ${ }^{6}$ Department of Brain and Cognitive Sciences, University of Rochester, Rochester, \\ New York 14627, and ${ }^{7}$ Center for Visual Science, University of Rochester, Rochester, New York 14627
}

Attention is a critical component of visual perception; however, the mechanisms of attention at the granular level are poorly understood. One possible mechanism by which attention modulates neuronal activity is to control the efficacy of communication between connected neurons; however, it is unclear whether attention alters communication efficacy across a variety of neuronal circuits. In parallel, attentional modulation of neuronal firing rate is not uniform but depends upon the match between neuronal feature selectivity and the feature required for successful task completion. Here we tested whether modulation of communication efficacy is a viable mechanism of attention by assessing whether it is consistent across a variety of neuronal circuits and dependent upon the type of information conveyed in each circuit. We identified monosynaptically connected pairs of V1 neurons through cross-correlation of neuronal spike trains recorded in adult female macaque monkeys performing attention-demanding contrast-change detection tasks. Attention toward the stimulus in the receptive field of recorded neurons significantly facilitated the efficacy of communication among connected pairs of V1 neurons. The amount of attentional enhancement depended upon neuronal physiology, with larger facilitation for circuits conveying information about task-relevant features. Furthermore, presynaptic activity was more determinant of attentional enhancement of communication efficacy than postsynaptic activity, and feedforward local circuits often displayed the largest facilitation with attention. Together, these findings highlight attentional modulation of communication efficacy as a generalized mechanism of attention and demonstrate that attentional modulation at the granular level depends on the relevance of feature-specific information conveyed by neuronal circuits.

Key words: attention; efficacy; monosynaptic connection; receptive field properties; V1

Significance Statement

How we pay attention to objects and locations in the visual environment has a profound impact on visual perception. Individual neurons in the visual cortex are similarly regulated by shifts in visual attention; however, the rules that govern whether and how attention alters neuronal activity are not known. In this study, we explored whether attention regulates communication between connected pairs of neurons in the primary visual cortex. We observed robust attentional facilitation of communication among these circuits. Furthermore, the extent to which the circuits were facilitated by attention depended on whether the information they conveyed was relevant for the particular attention task.

\section{Introduction}

Correct allocation of visual attention is an important component of visual perception. Much is known about the consequences of

Received Aug. 22, 2018; revised Oct. 29, 2018; accepted Nov. 17, 2018.

Author contributions: J.R.H.-S., W.M.U., and F.B. edited the paper; F.B. wrote the first draft of the paper; J.R.H.-S., W.M.U., and F.B. designed research; J.R.H.-S., V.L.M., and F.B. performed research; J.R.H.-S. and F.B. analyzed data; F.B. wrote the paper.

This work was supported by National Institutes of Health Grants NEl: EY013588 and EY012576 to W.M.U., EY018683 and EY025219 to F.B., and EY023165 to J.R.H.-S., National Science Foundation EPSCoR 1632738, the Whitehall Foundation, and the Hitchcock Foundation. V.L.M. was supported by Albert J. Ryan Foundation Graduate shifting the focus of attention on the activity of individual neurons (e.g., Moran and Desimone, 1985; Motter, 1993; Treue and Martínez Trujillo, 1999; Maunsell, 2015); however, the underlying neuronal mechanisms of attention remain unknown. More recently, it is becoming clear that many of the common readouts

Fellowship. We thank Elise Bragg for expert technical assistance; and Drs. Karen Moodie and Kirk Maurer for veterinary assistance.

The authors declare no competing financial interests.

Correspondence should be addressed to Farran Briggs at farran_briggs@urmc.rochester.edu.

https://doi.org/10.1523/JNEUROSCI.2164-18.2018

Copyright $\odot 2019$ the authors $\quad 0270-6474 / 19 / 391066-11 \$ 15.00 / 0$ 
of attention, such as changes in neuronal firing rates or correlated variability among neighboring neurons, vary substantially even within a visual cortical area (Nandy et al., 2017) and are not always related to subjects' behavior (Zénon and Krauzlis, 2012). Given that attentional modulation of neuronal activity is not uniform, it is critical to develop a more granular-level understanding of the rules that govern whether and how attention alters the activity of individual neurons. Such granular-level understanding may also provide a critical platform for elucidating the neuronal mechanisms of attention.

Previously, we put forward the hypothesis that one possible mechanism of attention involves facilitation of the efficacy of communication in neuronal circuits. This notion is based on the observation that attention enhances synaptic efficacy in geniculocortical circuits linking the LGN to primary visual cortex (V1) in the feedforward direction (Briggs et al., 2013). We proposed that attention could alter neuronal activity by dynamically reweighting synapses to selectively facilitate neuronal circuits carrying independent, stimulus- or task-relevant information and suppress circuits carrying redundant or irrelevant information. Under this regimen, the signal-to-noise ratio (SNR) for relevant visual information is increased in a manner that is independent of changes in neuronal firing rate. Accordingly, we observed a large increase in SNR at the level of V1 inputs even though attentional modulation of neuronal firing rate was modest (Briggs et al., 2013), consistent with many prior accounts (Motter, 1993; Luck et al., 1997; Mehta et al., 2000; McAdams and Reid, 2005; Yoshor et al., 2007). While we provided compelling evidence that facilitation of communication efficacy in neuronal circuits is a plausible mechanism of attention, these results were limited to geniculocortical circuits.

Attentional modulation of visual cortical neuronal activity is not uniform but varies substantially both within and across visual cortical areas (see, e.g., Motter, 1993; Luck et al., 1997; Mehta et al., 2000). We explained the source of some of this variability among V1 neurons by demonstrating correlations between attentional modulation of neuronal firing rate and physiological response properties of individual neurons (Hembrook-Short et al., 2017). Specifically, we found that attention facilitated activity in V1 neurons that were best tuned to the features that were relevant for successful completion of the attention task and suppressed activity in neurons tuned for orthogonal stimulus features. Given the dependence of attentional modulation among individual neurons on neuronal feature selectivity, any viable mechanism of attention must also account for this relationship.

Our overarching objective in this study was to determine whether facilitation of communication efficacy among neuronal circuits is a viable and generalizable mechanism of attention. Toward this objective, we first explored whether attentional enhancement of communication efficacy generalized across visual circuits, in particular to local cortical circuits within V1. We then examined whether attentional modulation of communication efficacy depended on the feature selectivity of connected neurons. We identified monosynaptically connected pairs of V1 neurons based on spiking cross-correlations, including pairs connected via excitatory or inhibitory synapses as well as pairs receiving common presynaptic input. We observed consistent increases in efficacy with attention for connected pairs, quantified as the change in cross-correlation peak area. We also observed a number of interesting patterns based on interactions between attentional modulation of communication efficacy and neuronal physiology. Broadly, these results supported the feature selectivity hypothesis (e.g., Martínez Trujillo and Treue, 2004), whereby attention most strongly facilitated local circuit interactions when neurons were selective for stimulus features that were relevant for successful task completion. Additionally, attentional facilitation of communication efficacy was larger for feedforward local circuits, providing a plausible mechanistic explanation for the hierarchical progression of attentional modulation of firing rate observed throughout the visual cortex.

\section{Materials and Methods}

This study involved new analyses of data collected as part of a previous study of attentional modulation of firing rate among single neurons recorded across the cortical layers of V1 (Hembrook-Short et al., 2017). Data were collected from 3 adult female macaque monkeys (Macacca mulatta), and all of the procedures performed during the prior study conformed to the guidelines set forth by the National Institutes of Health and were approved by the Institutional Animal Care and Use Committees at the Geisel School of Medicine at Dartmouth and the University of California, Davis. Surgical preparation, recording procedures, visual stimulation, eye tracking, behavioral task design, and behavioral results have been described in detail previously (Hembrook-Short et al., 2017).

Briefly, monkeys were equipped with head posts and recording chambers to enable recording access to V1. Monkeys were trained to maintain central fixation while drifting sinusoidal grating stimuli were displayed on a monitor. Monkeys performed fixation-only tasks in which they maintained central fixation while drifting gratings varying in contrast, orientation, spatial frequency, temporal frequency, or size were displayed within recorded neuronal receptive fields to generate neuronal tuning data. During the same recording sessions, monkeys performed a contrast-change detection task requiring covert shifts in visual spatial attention, following a Posner-cueing paradigm (Posner et al., 1980). On alternating blocks of trials (blocks contained 5-30 trials each), monkeys shifted spatial attention toward or away from the grating stimulus overlapping the receptive fields of recorded neurons to generate neuronal responses to stimuli in attend-toward and attend-away conditions. Monkeys were required to maintain central fixation within a small error window $(<0.35$ degrees) throughout the full duration of each trial to minimize the potential impact of small eye movements. Attention trials included a 0.3 s cue period followed by a $1-3 \mathrm{~s}$ visual stimulus display period (duration randomized per trial according to a hazard function with a mean of $1.7 \mathrm{~s}$ ) after which the contrast of one of the gratings increased by $10 \%$ and monkeys then had $1 \mathrm{~s}$ in which to answer that they detected this stimulus feature change by moving a joystick or pressing a button. Data were analyzed from sessions in which monkeys completed at least 40 correct trials per attention condition (for details, see Hembrook-Short et al., 2017).

Defining single units. Multiple V1 neurons were simultaneously recorded using concentric multielectrode arrays (Mini-Matrix array, Thomas Recording) or linear multielectrode arrays (U-Probe, Plexon) inserted daily into V1. Laminar positions of recorded neurons were determined per session based on proximity to orthodromically stimulated geniculocortical recipient neurons (Briggs et al., 2013) or relative to the layer $4 \mathrm{C} / 5$ border determined from local field potential responses to flashed stimuli (Hembrook-Short et al., 2017). Single-unit spike sorting methods, based on principal components analysis, were used to cluster and separate single-unit neuronal activity from noise. An SNR analysis was performed on spike waveforms (Kelly et al., 2007), and single units with SNRs $>2.75$ were included for further analysis. Additional criteria for inclusion of well-isolated single units were $<0.1 \%$ short interspike interval $(<1.2 \mathrm{~ms})$ violations and tuned responses to drifting gratings.

Spike train cross-correlations. New analyses were performed on data from recording sessions in which two or more well-isolated single units were recorded simultaneously. All analyses were performed using custom-written code (MATLAB, MathWorks). First, connected pairs of V1 neurons were identified by cross-correlating the spike trains of all possible pairings of simultaneously recorded, well-isolated V1 neurons. Spikes included for analysis were those recorded during the last four complete grating cycles ( $4 \mathrm{~Hz}$ stimulus, $1 \mathrm{~s}$ duration) before the grating contrast change in each correctly completed attention task trial. Spike 
trains were generated from attend-toward and attend-away trials and analyzed separately. Spike trains were binned at $1 \mathrm{~ms}$. Spike train crosscorrelations within $100 \mathrm{~ms}$ windows were computed per trial. For each pairing, cross-correlations were summed together for all trials of the same type (attend-toward or attend-away) recorded in the session. Neurons in each pair were designated as presynaptic or postsynaptic according to the sign of the cross-correlation peak latency. Summed raw cross-correlations were divided by the total number of spikes from the presynaptic neuron such that cross-correlations were expressed as percentages of total spikes (e.g., normalized). Shuffled cross-correlations were computed per trial by cross-correlating the same spike train from the presynaptic neuron with the spike train of the postsynaptic neuron shifted forward by one grating cycle $(-250 \mathrm{~ms})$. Shuffled crosscorrelations were summed together for trials of the same type and divided by the total number of spikes from the presynaptic neuron. For each pairing, the summed and normalized shuffled cross-correlation was then subtracted from the summed and normalized raw cross-correlation to produce a shuffle-corrected cross-correlation.

The latency of each shuffle-corrected cross-correlation peak was determined as the shift from zero (in milliseconds) of the maximum of the shuffle-corrected cross-correlation. Pairs of V1 neurons with crosscorrelation peaks centered at zero (zero-latency) putatively received common presynaptic input (Dan et al., 1998). Pairs of V1 neurons with cross-correlation peaks shifted away from zero (non-zero latency) were putative monosynaptically connected pairs (Reid and Alonso, 1995; Alonso and Martinez, 1998). For both common-input pairs and monosynaptically connected pairs, latencies for shuffle-corrected crosscorrelation peaks were identical across attention conditions because the latency is determined by fixed synaptic connections. Additionally, for each pair, the vertical separation distance between neurons was determined from the recording contact positions on the array.

Criteria for defining connected pairs. Pairs of V1 neurons were classified as connected pairs if all of the following criteria were met: (1) peaks in shuffle-corrected cross-correlations had to be sharp and narrow $(<5 \mathrm{~ms}$ full width at half-height) and an order of magnitude narrower than the onset response full width measured from neuronal peristimulus time histograms; (2) in at least one attention condition, the peak had to be $>2$ SDs above (or below in the case of negative amplitude peaks) the mean firing rate within the $100 \mathrm{~ms}$ window; and (3) in at least one attention condition, the peak had to reach or exceed $1 \%$ of total spikes. Pairs that met all of these criteria were further classified as follows. Pairs with peaks centered at zero (zero-latency peak pairs) were only included for further analysis if the neurons in the pair were separated by $>100 \mu \mathrm{m}$ vertical separation distance. In other words, neurons in the pair could not be on the same or immediately adjacent recording contacts of the array to ensure that spikes were from different neurons. Pairs with non-zero latency peaks were only included for further analysis if cross-correlation peaks were shifted away from zero and were asymmetrically shaped (Alonso and Martinez, 1998). Pairs with non-zero latency peaks could have positive or negative amplitude peaks, but in all cases, the inclusion criteria had to be met. Pairs of V1 neurons recorded on adjacent contacts or with $\leq 100 \mu \mathrm{m}$ vertical separation distance that displayed crosscorrelation peaks with latencies between 0 and $1 \mathrm{~ms}$ were classified as ambiguous pairs and were not included in final analyses because peaks may have been due to a mixture of common presynaptic input, monosynaptic connectivity, or potential cross talk across recording contacts.

From Monkey E (linear array recordings), 28 sessions were analyzed, including 255 single units with 1201 possible pairings. From those, 23 pairs with positive or negative amplitude, non-zero-latency crosscorrelation peaks were identified, for a hit rate of $2 \%$. Also, from Monkey E, 42 zero-latency cross-correlation peak pairs were identified, for a hit rate of 3.5\%. From Monkey B (concentric array recordings), 9 sessions were analyzed, including 26 single units with 27 possible pairings. From those, 9 pairs with positive amplitude, non-zero-latency crosscorrelation peaks were identified, for a hit rate of $33 \%$. Also, from Monkey B, 2 zero-latency cross-correlation peak pairs were identified, for a hit rate of $7 \%$. From Monkey $\mathrm{O}$ (concentric array recordings), 3 sessions were analyzed, including 8 single units with 10 possible pairings. From those, 2 pairs with positive amplitude, non-zero-latency cross- correlation peaks were identified, for a hit rate of $20 \%$. No zero-latency cross-correlation peak pairs were identified from Monkey O $(0 \%$ hit rate). Higher hit rates for Monkeys $\mathrm{B}$ and $\mathrm{O}$ were due to the type of multielectrode arrays used: electrodes in the concentric arrays were independently movable and were positioned to optimize potential connectivity (e.g., one electrode in layer $4 \mathrm{C}$ and one in layer 4B). Notably, although different types of electrodes were used in the 3 monkeys (concentric vs linear configurations; quartz-platinum/tungsten metal electrodes vs silicone contacts), there were no differences in attentional modulation of synaptic efficacy among pairs recorded in the 3 monkeys $\left(p=0.1, \mathrm{df}=(2,26), \chi^{2}=5.7 ;\right.$ Kruskal-Wallis test $)$. Therefore, data were pooled across monkeys. A total of 34 pairs with non-zero-latency cross-correlation peaks and 44 pairs with zero-latency cross-correlation peaks were analyzed further.

Experimental design and statistical analysis: attentional modulation of synaptic efficacy and tuning metric analyses. For each pair, attentional modulation of synaptic efficacy was measured as the change in crosscorrelation peak area across attention conditions. Peak area was calculated as the integral (width $= \pm 1 \mathrm{~ms}$ from peak center) of the shufflecorrected cross-correlation peak. Peak areas were calculated separately from shuffle-corrected cross-correlations measured from attend-toward and attend-away trials (by definition, peaks were centered at the same latency in both attention conditions). The change in peak area with attention was simply the difference in peak area across attention conditions (attend-toward peak area - attend-away peak area). Wilcoxon rank sum statistical tests were used to determine differences in shuffle-corrected cross-correlation peak area across attention conditions for positive amplitude peak pairs, negative amplitude peak pairs, and zero-latency peak pairs. Wilcoxon tests were also used to test for differences in peak latency and vertical separation distance between positive and negative amplitude peak pairs.

The following tuning metrics were computed for each neuron in a pair: orientation half-width at half-height $(\mathrm{HWHH})$, direction selectivity index, contrast to evoke a half-maximal response (c50), preferred spatial and temporal frequency, and surround suppression index (SSI) as described previously (Hembrook-Short et al., 2017). Each neuron in a pair was defined as Simple or Complex based on the f1/f0 ratio (Skottun et al., 1991). Attention modulation index values were calculated for each neuron in a pair as the difference divided by the sum of the average firing rate in each attention condition (Hembrook-Short et al., 2017). For 23 positive amplitude peak pairs and 2 negative amplitude peak pairs, a full set of tuning metrics (all tuning metrics except SSI) was available for both neurons in the pair. Additional pairs had partial tuning metrics for both neurons in the pair, including 16 pairs with size tuning data. Wilcoxon tests were used to test for differences in tuning metrics between presynaptic and postsynaptic neurons per pair. Across the sample of paired V1 neurons, presynaptic and postsynaptic V1 neurons displayed similar tuning for orientation, direction, contrast, spatial and temporal frequency, and size, and had similar $\mathrm{fl} / \mathrm{f} 0$ ratios ( $p$ value range $=0.2-0.9, z$ statistic range $=-0.2$ to 1.2 , Wilcoxon tests). To perform two-way comparisons of changes in cross-correlation peak area with attention and differential tuning metrics per pair (e.g., difference in $\mathrm{fl} / \mathrm{f} 0$, difference in $\mathrm{HWHH}$, difference in c50 for neurons per pair), linear regression analyses with statistical indicators $\left(R^{2}, p\right.$ values $)$ were used. To perform threeway comparisons of changes in cross-correlation peak area with attention and attention index (AI) values, $\mathrm{fl} / \mathrm{f} 0$ values, or tuning metrics for each neuron in a pair, 3D regression analyses with polynomial fits and statistical indicators $\left(R^{2}, \mathrm{p}\right.$ values) were used.

An additional 31 ambiguous pairs were identified as described above (23 pairs from Monkey E, 6 pairs from Monkey B, and 2 pairs from Monkey O). Analysis of changes in cross-correlation peak area with attention for these ambiguous pairs yielded a significant enhancement of synaptic efficacy with attention for ambiguous pairs $(p=0.0096, z$ statistic $=2.6$, Wilcoxon test; average cross-correlation peak area, attendtoward $=2.98 \pm 0.3$, average cross-correlation peak area, attend-away $=$ $1.98 \pm 0.4$ ), similar to the attentional enhancement of synaptic efficacy observed for positive peak pairs (see Fig. $2 A$ ). 
Data and code accessibility. Data and custom code that support the findings of this study are available from the corresponding author upon request.

\section{Results}

Our goal in this study was to determine whether facilitation of communication efficacy among neuronal circuits is a viable and generalizable neuronal mechanism of attention. Our previous work provided evidence in support of this idea, although these findings were limited to geniculocortical circuits (Briggs et al., 2013). In parallel, we found that attentional modulation of firing rate among V1 neurons depends on their visual physiology (Hembrook-Short et al., 2017). We therefore asked the following: (1) whether attention enhances synaptic efficacy among pairs of connected neurons within V1; and (2) whether attentional modulation of synaptic efficacy depends on the feature selectivity of connected neurons or the information conveyed by local circuits. Satisfying these two criteria would provide further compelling evidence to support the idea that selective modulation of communication efficacy in neuronal circuits is a generalizable mechanism of attention.

We performed novel analyses of data collected as a part of a prior study (Hembrook-Short et al., 2017). We used multielectrode arrays to record simultaneously from multiple, wellisolated single neurons in V1 in 3 alert macaque monkeys performing an attention-demanding contrast-change detection task (Fig. 1A). We cross-correlated the spike trains of all possible pairings of simultaneously recorded V1 neurons and identified connected pairs based on a number of strict criteria, including the following: cross-correlation peak height, width, and shape; consistency of cross-correlation peak latency across attention conditions; and vertical separation distance between recording contacts (see Materials and Methods). From 3 monkeys, we identified 34 pairs of V1 neurons with positive or negative amplitude cross-correlation peaks with non-zero-latencies. Our overall hit rate was $2.8 \%$ for recording putative monosynaptically connected pairs. From 2 monkeys, we identified 44 pairs of V1 neurons with zero-latency peaks, for an overall hit rate of $3.6 \%$ for recording putative pairs receiving common presynaptic input. Pairs with non-zero-latency peaks were classified based on asymmetric peak shape and peak latencies shifted away from zero. Examples of pairs of V1 neurons with non-zero-latency, positive amplitude cross-correlation peaks are illustrated in Figure $1 B$, and example pairs with non-zero-latency, negative amplitude cross-correlation peaks are illustrated in Figure $1 C$. Both positive and negative amplitude peaks suggested monosynaptic connectivity; however, negative amplitude cross-correlations indicated inhibition of the postsynaptic neuron. Cross-correlation peak latencies were consistent across attention conditions for all pairs (cross-correlations generated from attend-toward trials indicated in red, those generated from attend-away trials indicated in blue in Fig. 1). This was expected because synaptic connection delay times are physiologically fixed. Also noteworthy was the asymmetric shape of positive and negative amplitude, non-zerolatency cross-correlation peaks (Fig. $1 B, C$ ) compared with the symmetric shape of zero-latency cross-correlation peaks (examples illustrated in Fig. 1D). Zero-latency cross-correlation peaks also often displayed small negative amplitude dips on either side of the positive amplitude peak, possibly illustrating the refractory periods of the two neurons in the pair and/or the input neuron.

To examine whether attention modulated the efficacy of synaptic communication among local circuits within V1, we compared cross-correlation peak area across attention conditions for each identified connected pair of V1 neurons. We observed a significant increase in cross-correlation peak area with attention for pairs with positive amplitude peaks $(p=0.0018, z$ statistic $=$ 3.1, Wilcoxon test; Fig. $2 A$, left; for additional statistics, see Table 1). Indeed, all positive peak pairs demonstrated an increase in peak area with attention. Although negative cross-correlation peaks were slightly less negative with attention, peak area did not differ across attention conditions for V1 pairs with negative amplitude peaks ( $p=0.8$, no $z$ statistic due to small sample, Wilcoxon test; Fig. $2 A$, middle; Table 1). Attention did not influence peak area for zero-latency pairs, as most pairs displayed no change in peak area across attention conditions $(p=0.96, z$ statistic $=0.05$, Wilcoxon test; Fig. $2 A$, right; Table 1$)$.

Monosynaptically connected pairs of V1 neurons with positive or negative amplitude cross-correlation peaks differed from one another in two additional ways. First, cross-correlation peak latencies were significantly shorter for negative peak pairs ( $p=$ $0.026, z$ statistic $=2.2$, Wilcoxon test; Fig. $2 B$; Table 2 ), consistent with the notion that negative peak pairs were connected via faster inhibitory synapses (Ohana et al., 2012). Second, V1 neurons in negative peak pairs were separated by shorter distances compared with neurons in positive peak pairs $(p=0.032, z$ statistic $=-2.1$, Wilcoxon test; Fig. 2C; Table 2), consistent with the notion that inhibitory connections were often between proximal neurons (Stepanyants et al., 2009).

Having established that attention enhanced the efficacy of communication among pairs of V1 neurons with excitatory monosynaptic connections, we next explored whether attentional modulation of communication efficacy depended upon the physiological properties of neurons in each pair. First, we examined relationships between changes in cross-correlation peak area with attention and differences in physiological properties among neurons in each pair. We rationalized that differences in physiological properties among neurons per pair could serve as a proxy for the type of information communicated from presynaptic to postsynaptic neurons. We observed significant correlations between attentional modulation of communication efficacy and differences in $\mathrm{f} 1 / \mathrm{f} 0$ ratios $\left(R^{2}=0.39, p=0.04\right.$; Fig. $\left.3 A\right)$ and orientation HWHHs $\left(R^{2}=0.34, p=0.0015\right.$; Fig. $\left.3 B\right)$, but no significant correlation with difference in contrast sensitivity among neurons per pair $\left(R^{2}=0.12 ; p=0.2\right.$; Fig. $\left.3 C\right)$. More specifically, Simple-to-Complex neuron connections (positive $y$ values in Fig. $3 A$ ) were more enhanced with attention compared with Complex-to-Simple connections. Somewhat surprisingly, communication among pairs of connected V1 neurons was more facilitated by attention when the presynaptic neuron was more sharply tuned for orientation compared with the postsynaptic neuron (negative $y$ values in Fig. $3 B$ ). The relationship between attentional modulation of communication efficacy and differential orientation tuning could have been related to the fact that postsynaptic neurons per pair tended to be somewhat more direction selective, although direction selectivity did not differ significantly across presynaptic and postsynaptic neurons in this sample $(p=0.13, z$ statistic $=-1.5$, Wilcoxon test; Fig. $3 D)$. The lack of a significant interaction between attentional modulation of communication efficacy and differential contrast sensitivity was likely due to the fact that presynaptic and postsynaptic neurons had similar $c 50$ values $(p=0.21, z$ statistic $=1.2$, Wilcoxon test; Fig. 3E).

Next, we compared attentional modulation of communication efficacy with physiological properties reported for each neuron individually because differences in physiological properties may have masked nuanced interactions. Figure $4 A$ illustrates the 
A
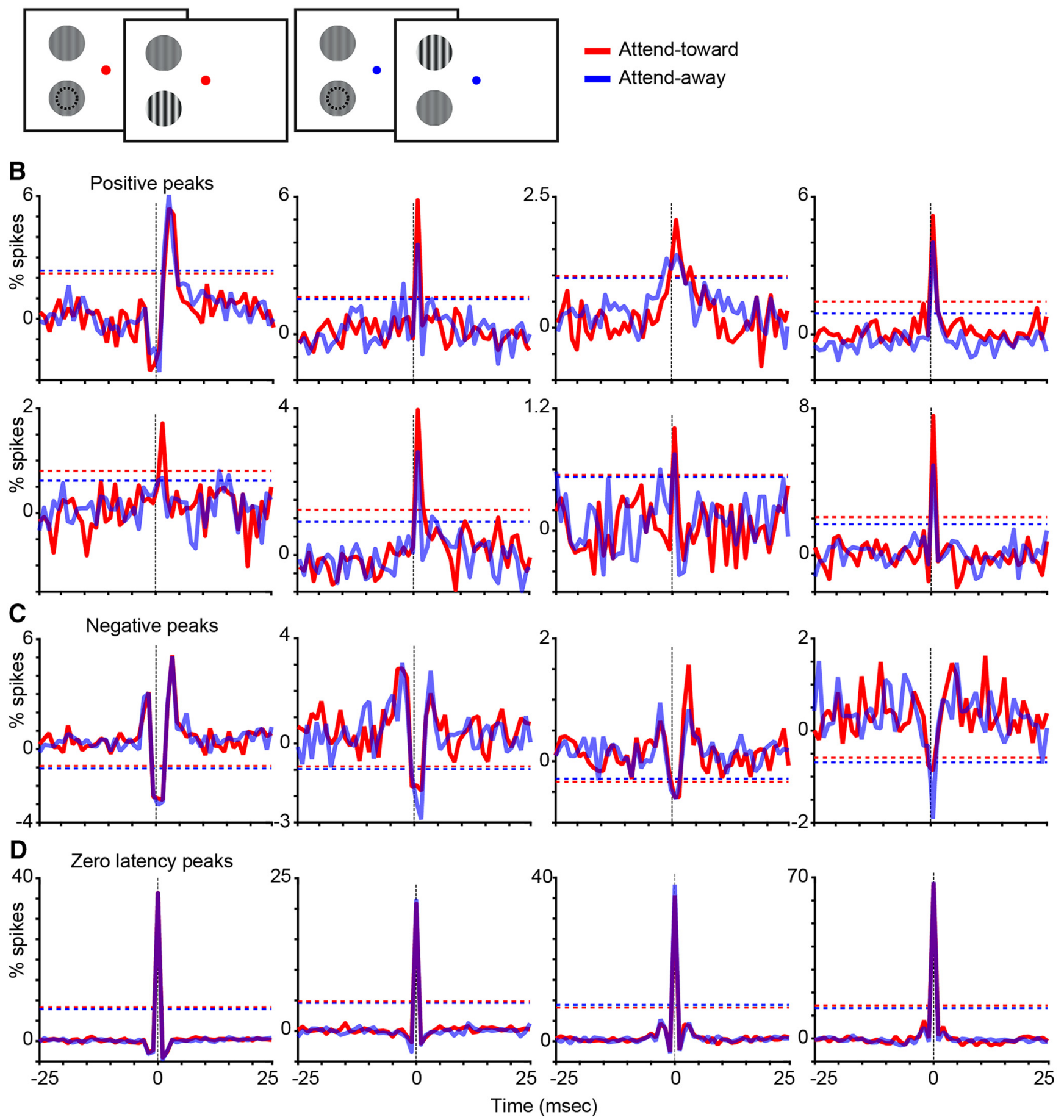

Figure 1. Attention task and examples of local circuit connectivity in V1.A, Schematic screen shots of the two conditions in the attention task. The color of the fixation dot cued monkeys to attend to the lower (red, attend-toward condition) or upper grating (blue, attend-away condition). Dashed circle (not displayed in task) represents the location of recorded neuronal receptive fields. Monkeys detected an increase in grating contrast that was validly cued on $95 \%$ of trials. B. Eight examples of monosynaptically connected pairs of V1 neurons with positive amplitude crosscorrelation peaks. Red represents spiking cross-correlations calculated from attend-toward trials. Blue represents spiking cross-correlations calculated from attend-away trials (made transparent). Red and blue dashed lines indicate 2SDs above the mean spike rate per attention condition. Vertical black dashed lines indicate zero latency. C, Four examples of monosynaptically connected pairs of V1 neurons with negative amplitude cross-correlation peaks. Conventions as in $\boldsymbol{B}$, except dashed red/blue lines indicate 2 SDs below mean spike rate. $\boldsymbol{D}$, Four examples of pairs of V1 neurons receiving common presynaptic input, indicated by cross-correlation peaks with zero latency. Conventions as in $\boldsymbol{B}$.

arrangement for these three-way comparisons with an illustration of the change in cross-correlation peak area with attention as a function of the laminar location of presynaptic and postsynaptic neurons per pair. Pairs are color coded according to the presynaptic and postsynaptic arrangement of Simple and Complex cells; open circles represent negative amplitude peak pairs, whereas filled circles represent positive amplitude peak pairs. As expected based on the laminar distribution of Simple and Complex cells in V1 (Gilbert and Wiesel, 1979; Martinez et al., 2002, 2005; Ringach et al., 2002), most Simple-to-Simple pairs (Fig. 4A, 


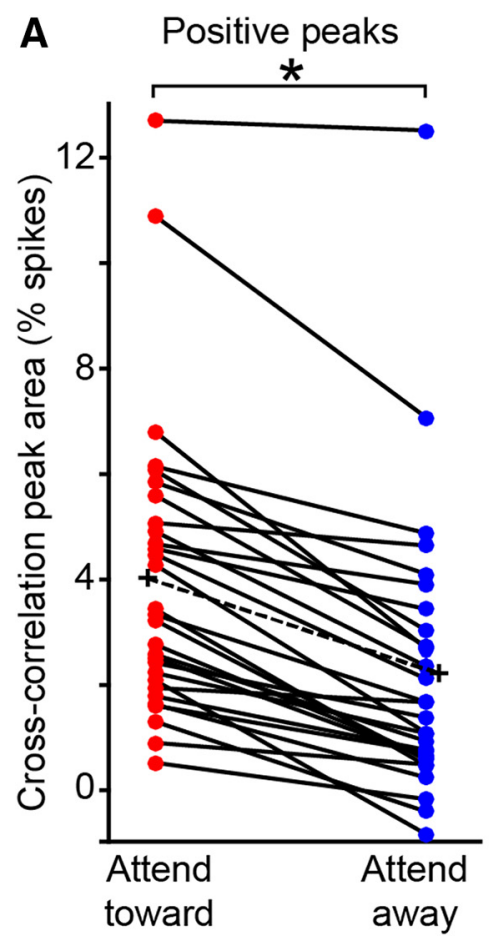

Negative peaks

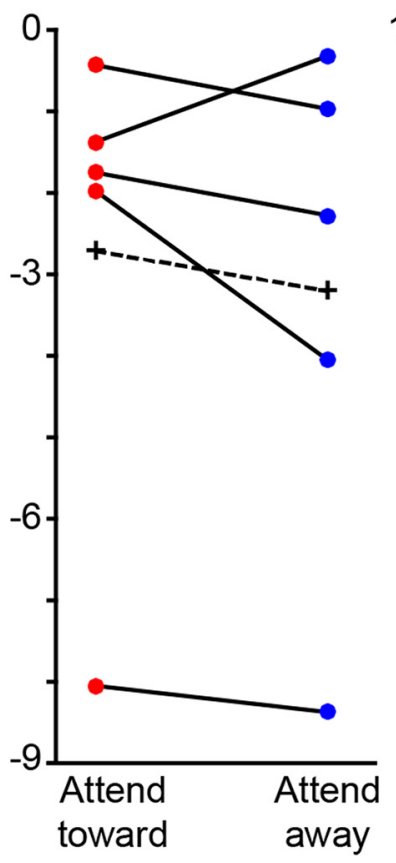

Zero latency peaks

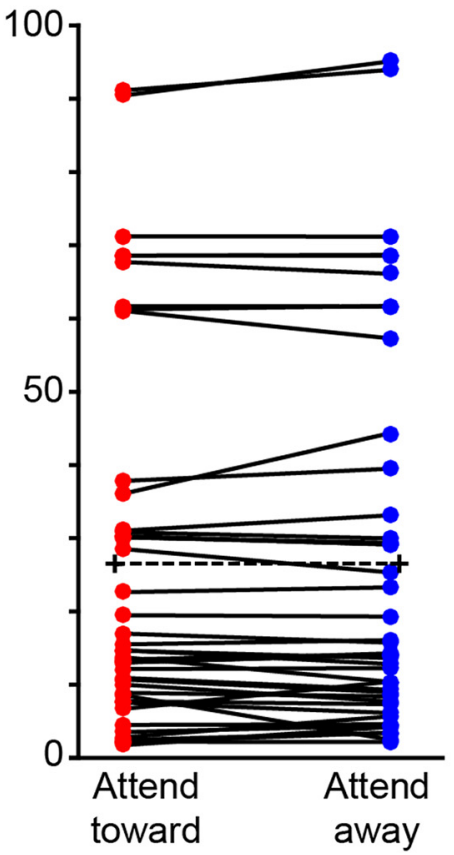

B
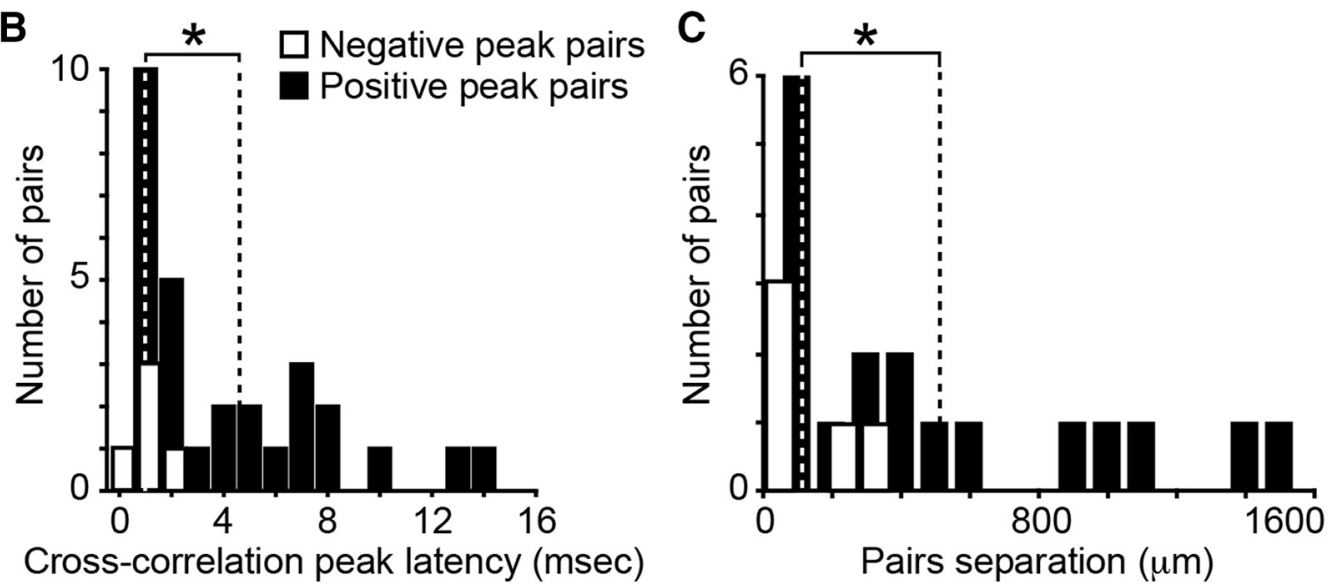

Figure 2. Attentional modulation of synaptic efficacy among connected V1 neurons. A, Spiking cross-correlation peak area measured from attend-toward (red dots) and attend-away (blue dots) trials for 29 positive peak pairs (left), 5 negative peak pairs (middle), and 44 zero-latency peak pairs (right). Black lines link data from the same pair across attention conditions. Plus signs linked by dashed lines indicate average cross-correlation peak area per sample and attention condition (Table 1). *Significant difference in cross-correlation peak area with attention for positive peak pairs $\left(p=0.0018, z\right.$ statistic $=3.1$, Wilcoxon test; Table 1). B. Distribution of cross-correlation peak latencies for positive peak pairs (black bars) and negative peak pairs (white bars). ${ }^{*}$ Significant difference in cross-correlation peak latency between positive and negative peak pairs $(p=0.026, z$ statistic $=2.2$, Wilcoxon test; Table 2). $C$, Distribution of vertical separation distance between neurons in each pair for positive (black bars) and negative peak pairs (white bars). ${ }^{*}$ Significant difference in separation distance for positive and negative peak pairs $(p=0.032, z$ statistic $=-2.1$, Wilcoxon test; Table 2).

Table 1. Attentional modulation of cross-correlation peak area for connected pairs

\begin{tabular}{cccc}
\hline & $\begin{array}{l}\text { Average cross-correlogram } \\
\text { peak area, Attend-toward } \\
\text { (\% spikes) }\end{array}$ & $\begin{array}{l}\text { Average cross-correlogram } \\
\text { peak area, Attend-away } \\
\text { (\% spikes) }\end{array}$ & $\begin{array}{l}p \text { value } \\
\text { (Wilcoxon } \\
\text { tests) }\end{array}$ \\
\hline $\begin{array}{c}\text { Positive peak } \\
\text { pairs }(n=29)\end{array}$ & $4.02 \pm 0.5$ & $2.2 \pm 0.5$ & 0.0018 \\
$\begin{array}{c}\text { Negative peak } \\
\text { pairs }(n=5)\end{array}$ & $-2.72 \pm 1.5$ & $-3.2 \pm 1.6$ & 0.8 \\
$\begin{array}{c}\text { Zero-latency } \\
\text { peak pairs } \\
(n=44)\end{array}$ & $26.6 \pm 3.8$ & $26.7 \pm 3.9$ & 0.96 \\
\hline
\end{tabular}

Table 2. Cross-correlation peak latency and recording contact separation distance for connected pairs

\begin{tabular}{lclll}
\hline & $\begin{array}{l}\text { Positive } \\
\text { peak pairs } \\
(n=29)\end{array}$ & $\begin{array}{l}\text { Negative } \\
\text { peak pairs } \\
(n=5)\end{array}$ & $\begin{array}{l}\text { Zero-latency } \\
\text { peak pairs } \\
(n=44)\end{array}$ & $\begin{array}{l}p \text { value } \\
\text { (Wilcoxon } \\
\text { tests })\end{array}$ \\
\hline $\begin{array}{c}\text { Average peak } \\
\text { latency (ms) }\end{array}$ & $4.2 \pm 0.7$ & $1.0 \pm 0.4$ & 0 & $0.026^{*}$ \\
$\begin{array}{c}\text { Average pairs } \\
\text { separation }(\mu \mathrm{m})\end{array}$ & $508.3 \pm 111$ & $130 \pm 58$ & $405 \pm 42$ & $0.032^{*}$ \\
\hline
\end{tabular}

*Statistical comparisons between positive and negative peak pairs only, as inclusion criteria were different for zero-latency pairs. 

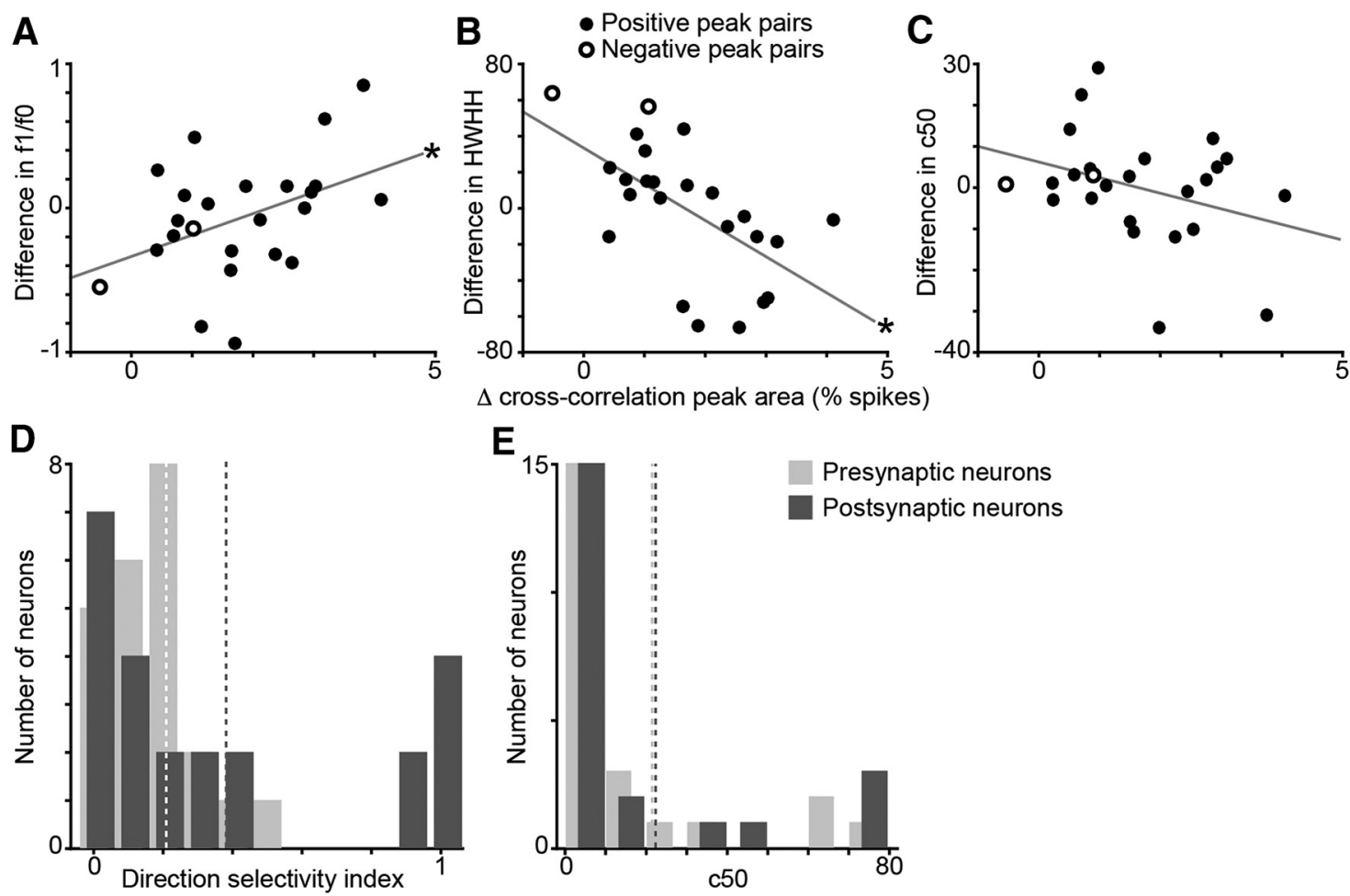

Figure 3. Relationships between attentional modulation of efficacy and comparative physiology of connected neurons. $\boldsymbol{A}$, Change in spiking cross-correlation peak area with attention as a function of the difference in $\mathrm{f} 1 / \mathrm{f0}$ for positive peak pairs (black dots) and negative peak pairs (white dots with black outline). Pairs with negative differential f1/f0 values are connected as Complex-to-Simple. Pairs with positive values are connected as Simple-to-Complex. Gray line indicates linear regression fit. *Significant correlation between differential f1/f0 and change in cross-correlation peak area $\left(R^{2}=0.39, p=0.04\right)$. $\boldsymbol{B}$, Change in cross-correlation peak area with attention as a function of the difference in HWHH for positive and negative peak pairs. Pairs with positive differential HWHH values are connected as less orientation tuned to more sharply tuned. Pairs with negative values are connected as more sharply tuned to less orientation tuned. Gray line indicates linear regression fit. *Significant correlation between differential HWHH and change in cross-correlation peak area $\left(R^{2}=0.34, p=0.0015\right)$. C, Change in cross-correlation peak area with attention as a function of the difference in 50 for positive and negative peak pairs. Pairs with positive differential $c 50$ values are connected as linear/low sensitivity to nonlinear/high sensitivity. Pairs with negative values are connected as nonlinear/high sensitivity to linear/low sensitivity. Gray line indicates linear regression fit $\left(R^{2}=0.12, p=0.2\right)$. D, Distributions of direction selectivity index values for presynaptic (light gray bars) and postsynaptic neurons (dark gray bars) per pair. Dark and light gray dashed lines indicate average direction selectivity values for each. $\boldsymbol{E}$, Distributions of c50 values for presynaptic and postsynaptic neurons per pair. Conventions as in $\boldsymbol{D}$. Dashed lines indicate that averages are overlapping.

green) were pairs in which both neurons were located in the granular laminar compartment, whereas most pairings involving neurons in the supragranular or infragranular laminar compartments included Complex cells (Fig. 4A, magenta, black, and orange). Notably, two Simple-to-Complex pairs in which the presynaptic neuron was in the granular laminar compartment and the postsynaptic neuron was in the supragranular laminar compartment (Fig. 4A, two rightmost magenta dots) displayed strong attentional facilitation of communication efficacy, suggesting that attention facilitated communication in "feedforward" local circuits.

There were many significant three-way interactions between attentional modulation of communication efficacy and physiological properties of individual neurons per pair. Not surprisingly, there was a significant correlation between attentional modulation of communication efficacy and AI values $\left(R^{2}=0.63\right.$, $p=2.8 \times 10^{-5}$; Fig. $4 B$ ). Most pairs in which individual neurons per pair displayed similar AI values (Fig. $4 B$, middle hump in curve) had intermediate levels of attentional facilitation of communication efficacy.

Attentional modulation of communication efficacy was also greater for pairs involving Simple cells, especially when Simple cells were presynaptic $\left(R^{2}=0.47, p=5.7 \times 10^{-5}\right.$; Fig. $\left.4 C\right)$, consistent with the differential f1/f0 results reported in Figure $3 A$. Together, these findings supported the notion that attention facilitated communication in "feedforward" local circuits that re- layed input-recipient signals to a second stage of local cortical processing.

Interestingly, attention facilitated communication efficacy more when at least one neuron per pair was relatively poorly tuned for orientation $\left(R^{2}=0.52, p=1.6 \times 10^{-5}\right.$; Fig. $\left.4 D\right)$, although attention modestly facilitated communication among pairs of Simple cells with intermediate orientation HWHHs (Fig. $4 D$, middle, front, green dots). These findings were also consistent with the differential orientation tuning results reported in Figure $3 B$. There was a nonsignificant trend whereby attentional facilitation of communication efficacy was greater when postsynaptic neurons displayed more direction selectivity than presynaptic neurons $\left(R^{2}=0.12, p=0.07\right.$; Fig. $\left.4 E\right)$, consistent with the observation that postsynaptic neurons tended to be more direction selective (Fig. 3D). These observations suggested that (1) attention facilitated communication among neuronal pairs with dissimilar orientation and/or direction tuning; and (2) the local circuits most strongly facilitated by attention were not optimized for refining orientation tuning (or direction selectivity) when monkeys performed a contrast-change detection task.

Although most neurons per pair displayed similar contrast sensitivity (Fig. 3E), attention facilitated communication efficacy more when the presynaptic neuron had a lower c50 compared with the postsynaptic neuron $\left(R^{2}=0.4, p=0.0003\right.$; Fig. $\left.4 F\right)$. Thus, attentional facilitation of local circuit communication appeared more driven by the contrast sensitivity of the presynaptic 

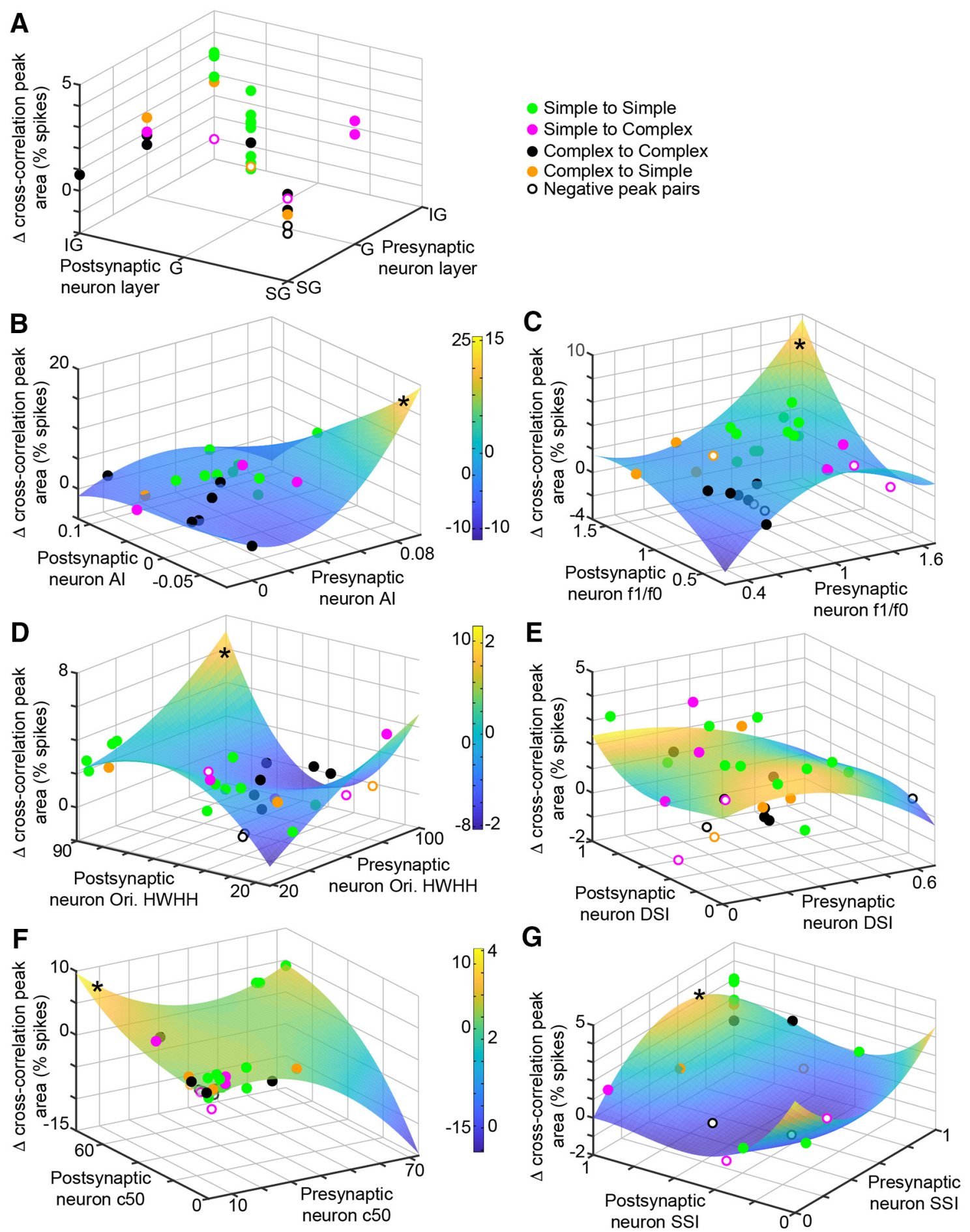

Figure 4. Relationships between attentional modulation of efficacy and physiology of individual neurons in each pair. A, 3D scatter plot illustrating the change in spiking cross-correlation peak area with attention and the sublaminar compartment location of presynaptic and postsynaptic neurons per connected pair. G, Granular; SG, supragranular; IG, infragranular compartment. Colors represent each Simple/Complex pairing. Closed dots represent positive peak pairs. Open dots represent negative peak pairs. B, Change in cross-correlation peak area with attention as a function of Al values for presynaptic and postsynaptic neurons per pair (each pair color-coded as in $A$ ). Colored manifold represents the $3 \mathrm{D}$ polynomial fit (scalebar middle, labels left indicate change in peak area with attention). ${ }^{*}$ Significant three-way interaction $\left(R^{2}=0.63, p=2.8 \times 10^{-5}\right)$. C, Change in cross-correlation peak area with attention as a function of f1/f0 ratios for presynaptic and postsynaptic neurons per pair. Colored manifold represents the 3D polynomial fit (scalebar middle, labels right, conventions as in $B)$. ${ }^{*}$ Significant three-way interaction $\left(R^{2}=0.47, p=5.7 \times\right.$ $\left.10^{-5}\right)$. D. Change in cross-correlation peak area with attention as a function of orientation HWHHs for presynaptic and postsynaptic neurons per pair. Colored manifold represents the 3D polynomial fit (scalebar middle, labels left, conventions as in $B$ ). ${ }^{*}$ Significant three-way interaction $\left(R^{2}=0.52, p=1.6 \times 10^{-5}\right)$. $\boldsymbol{E}$, Change in cross-correlation peak area with attention as a function of direction selectivity index (DSI) values for presynaptic and postsynaptic neurons per pair. Colored manifold represents the 3D polynomial fit (scalebar middle, labels right, conventions as in $B ; R^{2}=$ $0.12, p=0.07) . F$, Change in cross-correlation peak area with attention as a function of $\mathrm{c} 50$ values for presynaptic and postsynaptic neurons per pair. Colored manifold represents the $3 \mathrm{D}$ polynomial fit (scalebar middle, labels left, conventions as in $\boldsymbol{B})$. * Significant three-way interaction $\left(R^{2}=0.4, p=0.0003\right) . \mathbf{G}$, Change in cross-correlation peak area with attention as a function of $S S I$ values for presynaptic and postsynaptic neurons per pair. Colored manifold represents the $3 \mathrm{D}$ polynomial fit (scalebar middle, labels right, conventions as in $\boldsymbol{B})$. ${ }^{*}$ Significant three-way interaction $\left(R^{2}=\right.$ $0.71, p=4.1 \times 10^{-5}$ ). 
neuron. Because AI values tended to be higher for presynaptic neurons per pair (Fig. $4 B$ ) and neurons most sensitive to stimulus contrast were also most facilitated by attention (HembrookShort et al., 2017), it follows that attention facilitated local circuits in which the presynaptic neuron was more sensitive to contrast.

Contrary to most of the three-way interactions described above, the relationship between attentional modulation of communication efficacy and surround suppression was such that attentional facilitation was greatest when pairs of neurons displayed similar SSI values $\left(R^{2}=0.71, p=4.1 \times 10^{-5}\right.$; Fig. $\left.4 G\right)$. Interestingly, this relationship was consistent regardless of whether pairs of connected neurons shared low or high SSI values (Fig. 4G, yellow surfaces). This observation provided further support for the idea that attention facilitated local circuits that were not optimized to refine task-irrelevant stimulus features, such as size tuning, when monkeys were performing a contrast detection task.

\section{Discussion}

To determine whether facilitation of communication efficacy among neuronal circuits is a viable and generalizable mechanism of attention, we tested two predictions. First, we asked whether attentional facilitation of communication efficacy generalized across diverse neuronal circuits. Our observation of significant attentional enhancement of communication among V1 neurons supports the generality of this mechanism across early visual circuits. Second, we determined whether attentional modulation of synaptic efficacy depended on the feature selectivity of connected neurons or the information conveyed by local circuits. We discovered relationships between attentional modulation of local circuit interactions and the physiological properties of individual neurons in each connected pair. Together, our findings reveal that attentional modulation of neuronal activity, quantified as firing rate changes among individual neurons or alterations in communication efficacy among connected pairs of neurons, depends critically upon the types of visual signals conveyed by each neuron and circuit.

Previously, we used a stimulation approach to identify monosynaptic connections from LGN to V1. Because local stimulation (e.g., within V1) would have masked short-latency synaptic responses, we used a different approach to identify putative monosynaptically connected pairs of neurons within V1: crosscorrelation of spike trains of simultaneously recorded pairs of neurons (Fig. 1). Although this approach can yield false positives (Brody, 1999), it has been used with success to characterize connectivity and synaptic efficacy in early visual circuits (Nelson et al., 1992; Sillito et al., 1994; Reid and Alonso, 1995; Alonso and Martinez, 1998; Dan et al., 1998; Usrey et al., 2000; Alonso et al., 2001; Rathbun et al., 2010; Yu and Ferster, 2010; Vizuete et al., 2012). To avoid false positives, we applied a number of strict criteria to identify connected pairs of V1 neurons, following guidelines outlined previously (Reid and Alonso, 1995; Brody, 1998, 1999). Furthermore, we excluded "ambiguous" pairs defined by adjacent recording contacts and near-zero-latency crosscorrelation peaks. Interestingly, attention significantly facilitated communication efficacy among ambiguous pairs as it did for unambiguously defined pairs, suggesting that the attentional modulation of communication efficacy we observed was not confounded by false positive neuronal pairings.

We observed both positive amplitude and negative amplitude cross-correlation peaks among our sample of connected V1 neuronal pairs, indicating putative monosynaptic connectivity via excitatory (Reid and Alonso, 1995) and inhibitory synapses (Vi- zuete et al., 2012), respectively. Negative peak pairs had significantly shorter synaptic delay times (Fig. $2 B$ ), and neurons per pair were more proximal to one another (Fig. 2C) compared with positive peak pairs, further supporting connectivity via inhibitory synapses (Stepanyants et al., 2009; Ohana et al., 2012). Three pairs in our sample had cross-correlation peak latencies $>8 \mathrm{~ms}$, suggestive of possible disynaptic connectivity. However, percentages of correlated spikes and attentional modulation of communication efficacy for these three pairs were within the range of the sample.

Attention significantly enhanced the efficacy of communication among pairs of $\mathrm{V} 1$ neurons monosynaptically connected via excitatory synapses (Fig. 2A, left), consistent with our previous finding of attentional facilitation of geniculocortical inputs, which are also excitatory. This effect was quite robust as all positive amplitude peak pairs displayed an increase in peak area with attention. On average, attention reduced inhibition among negative peak pairs (Fig. 2A, middle); however, this effect was not statistically significant due to small sample size. Further study of attentional modulation of efficacy among inhibitory circuits will be essential to determine whether attention reduces inhibitory drive among local cortical circuits.

We observed no change in the efficacy of communication with attention for pairs of $\mathrm{V} 1$ neurons receiving common presynaptic input (Fig. $2 A$, right), consistent with our prior observation that attention did not facilitate communication efficacy among pairs of $\mathrm{V} 1$ neurons receiving divergent geniculocortical inputs (Briggs et al., 2013). These additional findings support our previous proposal that a reduction, or no change, in activity among redundant circuits may constitute a general mechanism by which attention reduces noise in sensory circuits. In other words, attention could selectively enhance activity and communication among neurons and circuits conveying independent and relevant sensory information and, at the same time, selectively reduce (or not change) activity among neurons receiving common, divergent input, leading to an SNR increase in visual circuits (Moran and Desimone, 1985).

We observed a number of significant interactions between attentional modulation of communication efficacy and the physiological properties of neurons per pair from which three general patterns emerged. In the first pattern, attentional facilitation of local circuit communication depended on the physiological properties of the presynaptic neuron. In the second pattern, local circuits most facilitated by attention were not optimized to refine non-task-relevant stimulus features. In the third pattern, attention most strongly facilitated local circuits conveying visual information in the feedforward direction.

The first general pattern we observed indicated that the physiological properties of the presynaptic neuron per pair often dictated the extent to which attention facilitated communication efficacy. For example, attentional facilitation of communication efficacy was greater when the presynaptic neuron was a Simple neuron and/or had a higher f1/f0 ratio compared with the postsynaptic neuron (Figs. $3 A, 4 C$ ). Circuit interactions involving two Complex cells were sometimes suppressed by attention (Fig. 4C, black dots). In parallel, attentional facilitation of communication efficacy was greater when the presynaptic neuron had a larger AI value (Fig. $4 B$ ). The positive relationship between attentional facilitation of communication efficacy and presynaptic neuronal AI value was not simply due to an increase in firing rate with attention because all cross-correlations were normalized by firing rate, and this normalization was computed separately for each attention condition. Additionally, AI values tend to be close to 
zero for V1 neurons (Motter, 1993; Luck et al., 1997; McAdams and Maunsell, 1999; Yoshor et al., 2007; Hembrook-Short et al., 2017). Thus, attentional modulation of communication efficacy may constitute a separate attention mechanism that is not yoked to attentional modulation of neuronal firing rate. Finally, attentional enhancement of communication efficacy was greater when the presynaptic neuron was more sensitive to changes in stimulus contrast compared with the postsynaptic neuron (Fig. $4 F$ ), even though c50 values were similar across presynaptic and postsynaptic neurons (Fig. 3E). Because monkeys were performing a contrast-change discrimination task and AI values correlated with contrast sensitivity (Hembrook-Short et al., 2017), logic dictates that attentional modulation of communication efficacy should also correlate with presynaptic neuronal contrast sensitivity.

The second pattern that emerged was a trend whereby attention facilitated local circuits that were not optimized to refine task-irrelevant stimulus features, such as orientation, direction, or size tuning. When orientation tuning and direction selectivity of connected neurons were examined, circuit interactions were more facilitated by attention when neurons had dissimilar tuning bandwidth for orientation or dissimilar direction selectivity. Specifically, attention facilitated V1 circuit interactions most when at least one neuron per pair had poor orientation tuning, usually the postsynaptic neuron (Figs. 3B, 4D). Although not significant, there was a trend whereby attentional facilitation of communication efficacy was greater when the postsynaptic neuron was more direction selective (Fig. 4E). In some respects, these observations are consistent with prior work demonstrating that attention does not alter feature tuning among visual cortical neurons (McAdams and Maunsell, 1999; Treue and Martínez Trujillo, 1999). Along similar lines, attentional facilitation of communication efficacy was larger when both neurons per pair had similar surround suppression index values, suggesting that the circuits most facilitated by attention were not processing new information about stimulus size or extraclassical interactions. Together, these findings support the notion that the local circuits most strongly facilitated by attention were not optimized for refining taskirrelevant stimulus features, such as orientation tuning, direction selectivity, or size selectivity, when monkeys were engaged in a contrast change detection task.

The third pattern that emerged provided new evidence, at the local circuit level, of a hierarchical progression of attentional modulation. There are numerous examples of progressive increases in attentional modulation of neuronal firing rate across hierarchically organized visual cortical areas (Motter, 1993; Luck et al., 1997; McAdams and Maunsell, 1999; Mehta et al., 2000; Gregoriou et al., 2009; Buffalo et al., 2010; Zhou and Desimone, 2011). Here, we showed that attentional modulation of communication efficacy within V1 was greater for putative feedforward circuits, in which Simple cells were presynaptic, compared with other local circuits (e.g., when Complex cells were presynaptic) (Figs. 3A, 4A,C). Indeed, some of the largest effects of attention on local circuit interactions occurred for Simple-to-Complex circuits connecting neurons in the granular laminar compartment with those in the supragranular compartment, representing a prominent component of the canonical local circuit map (Callaway, 2004; Douglas and Martin, 2004).

In conclusion, we discovered that attention enhanced the efficacy of communication in V1 local circuits, suggesting that attentional facilitation of communication efficacy is a general mechanism spanning early visual circuits. Attention enhanced communication efficacy in all monosynaptic excitatory circuits and reduced inhibition in all but one monosynaptic inhibitory circuit, although the sample size of inhibitory connections was too small to reach conclusions about the impact of attention on local inhibition. Correlated spiking among neurons receiving common presynaptic input was not altered by attention, supporting the notion that attention selectively boosts activity in circuits conveying independent, stimulus-relevant information over those conveying redundant and/or irrelevant information. Examination of interactions between attentional modulation of local circuit communication and neuronal physiology revealed a number of patterns. First, the physiological properties of presynaptic neurons determined the level of attentional facilitation of communication efficacy. Second, the local circuits most facilitated by attention were not optimized to sharpen task-irrelevant feature tuning, such as orientation tuning, direction selectivity, or surround suppression. Third, attentional facilitation of V1 local circuits was largest for feedforward circuits, in line with a hierarchical model of progressive attentional facilitation. Together, these findings suggest that attentional modulation of communication efficacy among local circuits is a generalized mechanism of attention that is driven by presynaptic activity and depends on the relevance of feature-specific information conveyed by each circuit.

\section{References}

Alonso JM, Martinez LM (1998) Functional connectivity between simple cells and complex cells in cat striate cortex. Nat Neurosci 1:395-403. CrossRef Medline

Alonso JM, Usrey WM, Reid RC (2001) Rules of connectivity between geniculate cells and simple cells in cat primary visual cortex. J Neurosci 21:4002-4015. CrossRef Medline

Briggs F, Mangun GR, Usrey WM (2013) Attention enhances synaptic efficacy and the signal-to-noise ratio in neural circuits. Nature 499:476-480. CrossRef Medline

Brody CD (1998) Slow covariations in neuronal resting potentials can lead to artefactual fast cross-correlations in their spike trains. J Neurophysiol 80:3345-3351. CrossRef Medline

Brody CD (1999) Correlations without synchrony. Neural Comput 11: 1537-1551. CrossRef Medline

Buffalo EA, Fries P, Landman R, Liang H, Desimone R (2010) A backward progression of attentional effects in the ventral stream. Proc Natl Acad Sci U S A 107:361-365. CrossRef Medline

Callaway EM (2004) Cell types and local circuits in primary visual cortex of the macaque monkey. In: The visual neurosciences (Chalupa L, Werner J, eds), pp 680-694. Cambridge, MA: Massachusetts Institute of Technology.

Dan Y, Alonso JM, Usrey WM, Reid RC (1998) Coding of visual information by precisely correlated spikes in the lateral geniculate nucleus. Nat Neurosci 1:501-507. CrossRef Medline

Douglas RJ, Martin KA (2004) Neuronal circuits of the neocortex. Annu Rev Neurosci 27:419-451. CrossRef Medline

Gilbert CD, Wiesel TN (1979) Morphology and intracortical projections of functionally characterized neurones in the cat visual cortex. Nature 280: 120-125. CrossRef Medline

Gregoriou GG, Gotts SJ, Zhou H, Desimone R (2009) High-frequency, long-range coupling between prefrontal and visual cortex during attention. Science 324:1207-1210. CrossRef Medline

Hembrook-Short JR, Mock VL, Briggs F (2017) Attentional modulation of neuronal activity depends on neuronal feature selectivity. Curr Biol 27: 1878-1887.e5 CrossRef Medline

Kelly RC, Smith MA, Samonds JM, Kohn A, Bonds AB, Movshon JA, Sing Lee TS (2007) Comparison of recordings from microelectrode arrays and single electrodes in the visual cortex. J Neurosci 27:261-264. CrossRef Medline

Luck SJ, Chelazzi L, Hillyard SA, Desimone R (1997) Neural mechanisms of spatial selective attention in area V1, V2, and V4 of macaque visual cortex. J Neurophysiol 77:24-42. CrossRef Medline

Martinez LM, Alonso JM, Reid RC, Hirsch JA (2002) Laminar processing of 
stimulus orientation in cat visual cortex. J Physiol 540:321-333. CrossRef Medline

Martinez LM, Wang Q, Reid RC, Pillai C, Alonso JM, Sommer FT, Hirsch JA (2005) Receptive field structure varies with layer in the primary visual cortex. Nat Neurosci 8:372-379. CrossRef Medline

Martínez Trujillo JC, Treue S (2004) Feature-based attention increases the selectivity of population responses in primate visual cortex. Curr Biol 14:744-751. CrossRef Medline

Maunsell JH (2015) Neuronal mechanisms of visual attention. Annu Rev Vis Sci 1:373-391. CrossRef Medline

McAdams CJ, Maunsell JH (1999) Effects of attention on orientationtuning functions of single neurons in macaque cortical area V4. J Neurosci 19:431-441. CrossRef Medline

McAdams CJ, Reid RC (2005) Attention modulates the responses of simple cells in monkey primary visual cortex. J Neurosci 25:11023-11033. CrossRef Medline

Mehta AD, Ulbert I, Schroeder CE (2000) Intermodal selective attention in monkeys: I. Distribution and timing of effects across visual areas. Cereb Cortex 10:343-358. CrossRef Medline

Moran J, Desimone R (1985) Selective attention gates visual processing in the extrastriate cortex. Science 229:782-784. CrossRef Medline

Motter BC (1993) Focal attention produces spatially selective processing in visual cortical areas V1, V2, and V4 in the presence of competing stimuli. J Neurophysiol 70:909-919. CrossRef Medline

Nandy AS, Nassi JJ, Reynolds JH (2017) Laminar organization of attentional modulation in macaque visual area V4. Neuron 93:235-246. CrossRef Medline

Nelson JI, Salin PA, Munk MH,Arzi M, Bullier J (1992) Spatial and temporal coherence in cortico-cortical connections: a cross-correlation study in areas 17 and 18 in the cat. Vis Neurosci 9:21-37. CrossRef Medline

Ohana O, Portner H, Martin KA (2012) Fast recruitment of recurrent inhibition in the cat visual cortex. PLoS One 7:e40601. CrossRef Medline

Posner MI, Synder CR, Davidson BJ (1980) Attention and the detection of signals. J Exp Psychol Gen 109:160-174. CrossRef Medline

Rathbun DL, Warland DK, Usrey WM (2010) Spike timing and informa- tion transmission at retinogeniculate synapses. J Neurosci 30:1355813566. CrossRef Medline

Reid RC, Alonso JM (1995) Specificity of monosynaptic connections from thalamus to visual cortex. Nature 378:281-284. CrossRef Medline

Ringach DL, Shapley RM, Hawken MJ (2002) Orientation selectivity in macaque V1: diversity and laminar dependence. J Neurosci 22:5639-5651. CrossRef Medline

Sillito AM, Jones HE, Gerstein GL, West DC (1994) Feature-linked synchronization of thalamic relay cell firing induced by feedback from the visual cortex. Nature 369:479-482. CrossRef Medline

Skottun BC, De Valois RL, Grosof DH, Movshon JA, Albrecht DG, Bonds AB (1991) Classifying simple and complex cells on the basis of response modulation. Vision Res 31:1079-1086. CrossRef Medline

Stepanyants A, Martinez LM, Ferecskó AS, Kisvárday ZF (2009) The fractions of short- and long-range connections in the visual cortex. Proc Natl Acad Sci U S A 106:3555-3560. CrossRef Medline

Treue S, Martínez Trujillo J (1999) Feature-based attention influences motion processing gain in macaque visual cortex. Nature 399:575-579. CrossRef Medline

Usrey WM, Alonso JM, Reid RC (2000) Synaptic interactions between thalamic inputs to simple cells in cat visual cortex. J Neurosci 20:5461-5467. CrossRef Medline

Vizuete JA, Pillay S, Diba K, Ropella KM, Hudetz AG (2012) Monosynaptic functional connectivity in cerebral cortex during wakefulness and under graded levels of anesthesia. Front Integr Neurosci 6:90. CrossRef Medline

Yoshor D, Ghose GM, Bosking WH, Sun P, Maunsell JH (2007) Spatial attention does not strongly modulate neuronal responses in early human visual cortex. J Neurosci 27:13205-13209. CrossRef Medline

Yu J, Ferster D (2010) Membrane potential synchrony in primary visual cortex during sensory stimulation. Neuron 68:1187-1201. CrossRef Medline

Zénon A, Krauzlis RJ (2012) Attention deficits without cortical neuronal deficits. Nature 489:434-437. CrossRef Medline

Zhou H, Desimone R (2011) Feature-based attention in the frontal eye field and area V4 during visual search. Neuron 70:1205-1217. CrossRef Medline 